\title{
A Correlation of FTIR Spectra Derived from Prostate Cancer Biopsies with Gleason Grade and Tumour Stage
}

\author{
Ehsan Gazi ${ }^{a, b, *}$, M. Baker ${ }^{a}$, J. Dwyer ${ }^{a}$, N.P. Lockyer ${ }^{a}$, P. Gardner ${ }^{a}$, J.H. Shanks ${ }^{c}$, \\ R.S. Reeve ${ }^{d}$, C.A. Hart ${ }^{b}$, N.W. Clarke ${ }^{b, e}$, M.D. Brown ${ }^{b}$ \\ a School of Chemical Engineering and Analytical Science, University of Manchester, Manchester, UK \\ ${ }^{\mathrm{b}}$ ProMPT Genito Urinary Cancer Research Group, Cancer Research United Kingomd, Paterson Institute, \\ Christie Hospital NHS Trust, Manchester, UK \\ ${ }^{\mathrm{c}}$ Histopathology Department, Christie Hospital NHS Trust, Manchester, UK \\ d Histopathology Department, Hope Hospital NHS Trust, Manchester, UK \\ e Department of Urology, Hope Hospital NHS Trust, Manchester, UK
}

\section{Article info}

Article history:

Accepted March 13, 2006

Published online ahead of

print on •••

\section{Keywords:}

Gleason grade

FTIR microspectroscopy

Molecular diagnosis

Multivariate analysis

Prostate cancer

Tumour stage

\begin{abstract}
Objectives: We introduce biochemistry as a second dimension to Gleason grading, using Fourier transform infrared (FTIR) microspectroscopy. For the first time, we correlate FTIR spectra derived from prostate cancer (pCA) tissue with Gleason score and the clinical stage of the tumour at time of biopsy.

Methods: Serial sections from paraffin-embedded pCA tissue were collected. One was stained with hematoxylin and eosin and Gleason scored; FTIR spectra were collected from malignant locations using a second unstained section. FTIR spectra, representing different Gleason grades, were used to construct a diagnostic classifier for pCA using linear discriminant analysis (LDA). This model was blind tested using 383 IR spectra from 36 biopsies.

Results: Using a three-band Gleason criteria, we obtained sensitivity of $\geq 70 \%$ for the FTIR-LDA model to predict Gleason $<7,=7$, and $>7$, with specificities of $\geq 81 \%$. Using a threshold of Gleason/FTIR-LDA score of $\geq 8$, we obtained a sensitivity and specificity of $71 \%$ and $67 \%$, respectively, for the correlation with metastatic tumours using the FTIR-LDA system and $85 \%$ and $63 \%$, respectively, for the correlation of metastatic tumours using the Gleason system.

Conclusions: There is a correlation between tissue architecture using Gleason score with tissue biochemistry using FTIR-LDA. Both systems are similar in their performance in predicting metastatic behaviour in tumours from individual patients.
\end{abstract}

C) 2006 Elsevier B.V. All rights reserved.

* Corresponding author. ProMPT Genito-Urinary Cancer Research, Cancer Research UK Paterson Institute, Christie Hospital NHS Trust, Wilmslow Road, Manchester, M20 4BX, UK. Tel. +44 161446 3232; Fax: +44 1614463109.

E-mail address: EGazi@picr.man.ac.uk (E. Gazi). 


\section{Introduction} grade [5].
Prostate tumour assessment of biopsy material is predominantly carried out by the method of Gleason grading [1]. However, a limitation in this technique is the disparity between tissue architecture and biochemical progression, particularly after hormone manipulation [2]. Also, difficulties concerning the reproducibility of a specific Gleason score, due to intraobserver and interobserver variability, mitigate its acceptance as a single prognostic tool $[3,4]$. Despite the problems with Gleason grading, the majority of studies based on prostate cancer (pCA) prognosis use this system and some authorities have suggested that all new prognostic markers should be evaluated in conjunction with Gleason

The concerns relating to Gleason grading have called for the development of diagnostic tools that operate on the basis of cellular biochemical rather than visual tissue architectural recognition. Vibrational microspectroscopy in the form of Fourier transform infrared (FTIR) and Raman spectroscopy, combined with imaging technology and multivariate statistical approaches to data analysis, have been much to the forefront in the development of practical diagnostic tools for the detection and cytopathologic grading of various neoplasms [6], such as pCA $[7,8-11,12]$. FTIR microspectroscopy measures the vibrational modes (mainly, stretching and bending) of functional groups of biomolecules as low-energy IR photons $(0.05-0.5 \mathrm{eV})$ are transmitted through the tissue, in vitro. The resulting IR spectrum is characteristic of the tissue's biochemical composition (lipid, protein, carbohydrate, and phosphorylated molecular domains).

Previously, our group has applied linear discriminant analysis (LDA) to FTIR spectra derived from paraffin-embedded pCA tissue of each Gleason grade (2-5), as a means of constructing an operator-independent diagnostic algorithm for pCA grading $[10,11]$. In the present study, we hypothesise that as the result of phenotypic heterogeneity within the population of malignant pCA cells, the FTIR-LDA grades generated from tumour lesions of pCAbiopsied tissue will differ from its associated Gleason grades. To test this hypothesis, we blind tested our FTIR-LDA diagnostic model with biopsies of known Gleason score and implemented analytical criteria that allow for the direct comparison of grades derived from each system. Secondly, we have correlated FTIR data, derived from tumour biopsies, with the clinical stage of these tumours (using the TNM system of prostate tumour classification) at the time of biopsy. Moreover, this correlation was used to investigate whether metastatic disease could be predicted through FTIR-LDA analyses.

\section{Materials and methods}

\subsection{Primary tissue preparation and sampling for FTIR}

Forty pCA tissue biopsy specimens were obtained as paraffinembedded blocks (Genito-Urinary Cancer Research Group, Paterson Institute) from patients with pCA. With the exception of three biopsies that were radical prostatectomies and one transrectal ultrasound of the prostate, all samples were obtained with informed consent from men undergoing treatment for urinary outflow obstructive symptoms by transurethral resection in which there was prostate adenocarcinoma, following a single evaluation. Serial sections were collected at $10 \mu \mathrm{m}$ thickness from each specimen, one of which was mounted onto $\mathrm{a} \mathrm{BaF}_{2}$ plate (Linkam Scientific) with the adjacent section mounted onto a glass slide and stained with hematoxylin and eosin (H\&E). An experienced histopathologist (J.H.S.) assigned Gleason scores to areas of malignancy identified within the H\&E sections. The complimentary sections, mounted onto $\mathrm{BaF}_{2}$ plates, were washed on an orbital mixer with Citroclear for $6 \mathrm{~min}$ to remove the paraffin and then acetone at $4{ }^{\circ} \mathrm{C}$ for a further $6 \mathrm{~min}$ before being air-dried for $1 \mathrm{~h}$ under ambient conditions. The anatomic features identified from the $H \& E$ section were used as landmarks to position the IR beam on the malignant lesions of the unstained adjacent section.

\subsection{FTIR microspectroscopy}

FTIR spectra of Gleason-graded primary prostate tissues were collected in transmission mode using a Nicolet Magna system 550 spectrometer equipped with a liquid nitrogen cooled MCT/A detector and a $\mathrm{KBr}$ beam splitter. The spectrometer was attached to a microscope equipped with a video camera to view optical images ( $\times 150$ magnification) of the sampling area and a programmable computerized $x-y$ stage. An aperture size of $60 \times 60 \mu \mathrm{m}$ was used to collect malignant epithelial cell spectra. A simplified diagram of the FTIR microscope is shown in Fig. 1. FTIR spectra represent an average of 512 scans in the mid-IR wavenumber range $750-4000 \mathrm{~cm}^{-1}$ with a spectral resolution of $4 \mathrm{~cm}^{-1}$. Background scans were obtained from a region of no sample and ratioed against the sample spectrum.

\subsection{Data processing}

IR spectra acquired from each Gleason-graded prostate tissue section were baseline corrected between the spectral region $\sim 1720 \mathrm{~cm}^{-1}$ to $\sim 980 \mathrm{~cm}^{-1}$ and normalised to the amide I $\left(\sim 1650 \mathrm{~cm}^{-1}\right)$ peak intensity, using the OMNIC v.5.1a software. Following this, the band region between $1481 \mathrm{~cm}^{-1}$ to $999 \mathrm{~cm}^{-1}$ (diagnostic spectral region) was used to construct or blind test the FTIR-LDA grading model using SPSS Release 11.0.0.

\subsection{Statistical analysis}

The Spearman rho, nonparametric (2-tailed) test was used to determine whether there was a significant correlation
76

77

78

79

80

81

82

83

84

85

86

87

88

89

90

91

92

93

94

95

96

97

98

99

100 


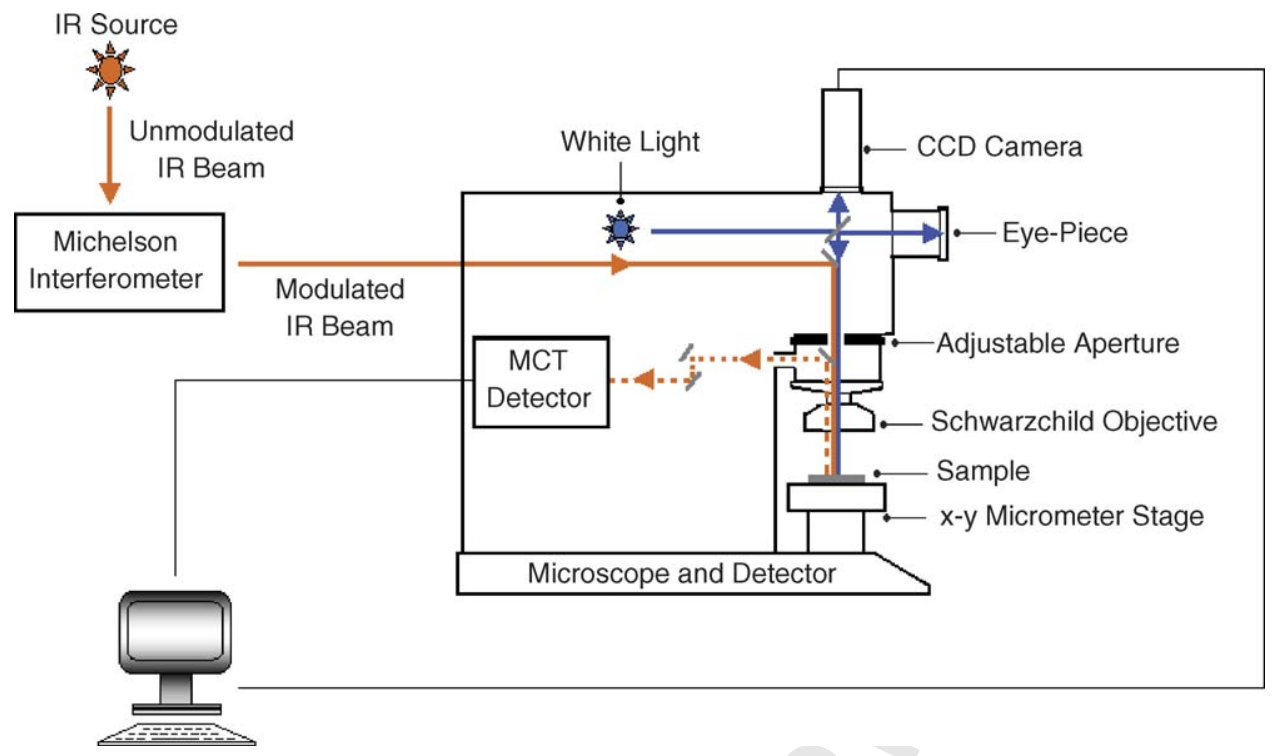

Fig. 1 - A simplified diagram of the Fournier transform infrared (FTIR) microscope system. Unmodulated polychromatic IR light, emitted as blackbody radiation from a silicon carbide glowbar, is directed into a Michelson interferometer. Here, a KBR beam splitter, combined with a movable and fixed mirror produces a modulated IR beam with a frequency low enough to be detected by the MCT detector. This IR beam is introduced into the FTIR microscope and transferred through mirrors and focussed by a $\times 15$ Schwarzchild objective towards the tissue sample (on a MirrIR substrate), which is placed on an $x-y$ micrometer stage. The tissue area illuminated by the incident IR beam (solid red line) is defined by an adjustable aperture. Conveniently, the IR beam is passed through the same optical train as the sample illumination light (white light; blue solid line), which together with the CCD camera, permits the capture of photomicrographs of the precise area analysed by IR. IR light is attenuated at specific frequencies after passing through the tissue. In reflection mode, this attenuated light (red dotted line) is reflected off the MirrIR substrate and passed back through the tissue and Schwarzchild objective. Approximately $40-50 \%$ of the attenuated IR light is blocked by a mirror that collects the reflected beam, which is subsequently directed to the MCT detector. The photovoltaic semiconductor MCT detector measures the IR signal in the time domain. These data are converted into the frequency domain through Fourier transform by a computer. For further information concerning the operation of the FTIR microscope, the reader is directed to Stuart [13].

between the FTIR-LDA and Gleason score derived from these blind-tested biopsies. The independent samples (2-tailed) $t$ test, at $95 \%$ confidence interval (CI), was used to test whether the FTIR-LDA or Gleason scores generated from low-stage tumours (T1 and T2) were significantly different from those of high-stage tumours (T3 and T4).

\section{Results}

\subsection{Construction of the FTIR-LDA pCA tumour-grading} model

The H\&E-stained tissue sections shown in Fig. 2 represent epithelial areas from which IR spectra were acquired and subsequently used to define the Gleason clusters in the FTIR-LDA grading model shown in Fig. 3A. The mean IR spectra derived from each spectral data set used to train the Gleason clusters in the LDA combined groups plot are presented in Fig. 3B and $C$.
LDA [14] permits the construction of a predictive model that can be used for multigroup data classification and dimensionality reduction for a given data set. The principles of this procedure are as follows. We consider a number of groups (IR spectra of each specimen of Gleason-graded tissue), each group consisting of a number of cases (intensities at each wavenumber). LDA generates a first discriminant function, based on a linear combination of predictor variables generated from each case that best separate the groups. A second discriminant function is generated, which is uncorrelated with function 1 and further separates the groups, the result of which allows maximal variance between groups and minimal variance within groups. Thus, a cluster plot (linear combined groups plot of discriminant function weights) is generated and can be considered as a prototype diagnostic classifier (the FTIR-LDA grading model in Fig. 3A). The initial group classification results 


\section{Increasing Biological Malignancy}
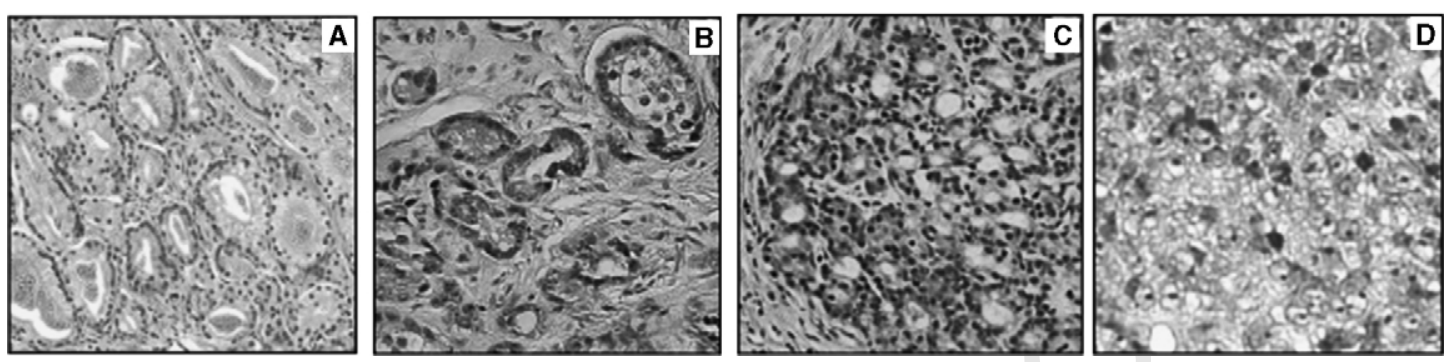

Fig. 2 - Hematoxylin and eosin-stained prostate cancer (pCA) tissue of Gleason grades 2-5, exhibiting examples of malignant glands that were used to acquire training infrared spectra for the construction of the Fournier transform infrared-linear discriminant analysis grading model. (A) Gleason grade 2 pCA, (B) Gleason grade 3 pCA, (C) Gleason grade 4 pCA, (D) Gleason grade 5 pCA.

for IR spectra in the FTIR-LDA-grading model is shown in Table 1.

\subsection{Correlating biospectroscopic FTIR-LDA score to} histologic Gleason score

A blind test was carried out to assess the performance of the FTIR-LDA-grading model with regard to whether the clusters generated in this model were representative of each Gleason-graded disease state. In the blind test, 383 IR spectra were recorded from 40 individual tumour locations within Gleasongraded pCA tissue, which, in turn, were taken from 36 biopsies of different patients with pCA, whose tumours were not used to define any grades in the training data set. IR spectra used in the blind test

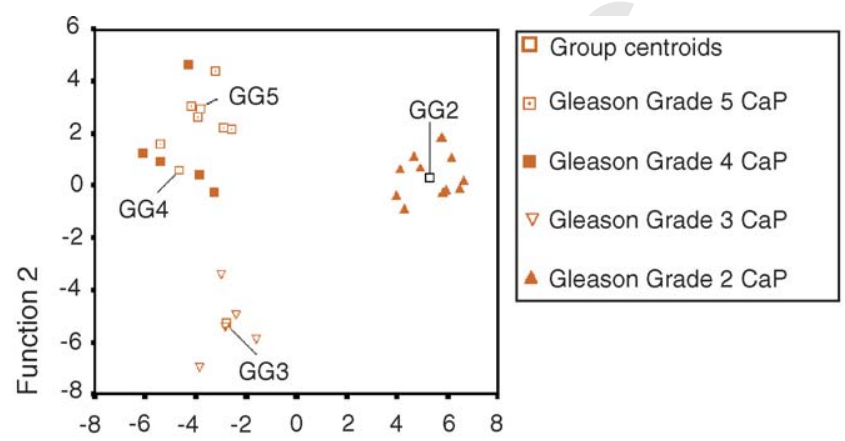

(A) Function 1
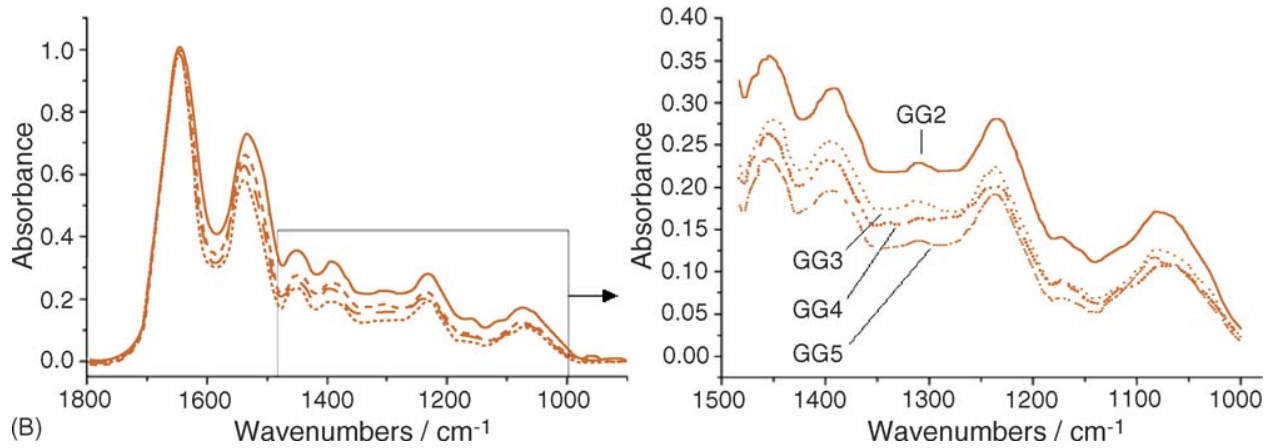

Fig. 3 - (A) Combined-groups plot of linear discriminant function weights for Fournier transform infrared (FTIR) spectra taken from prostate epithelial cells of Gleason-graded malignant states in primary prostate tissue (the FTIR-linear discriminant analysis [LDA] tumour-grading model); (B) Mean IR spectra recorded from malignant epithelial cells of different Gleason-graded (GG) 2-5 tissue. Boxed area represents the IR diagnostic spectral patterns in band region $1480 \mathrm{~cm}^{-1}$ to $1000 \mathrm{~cm}^{-1}$ used to train the FTIR-LDA grading model. 
Table 1 - Discriminant analysis classification results of IR spectra used to construct the FTIR-LDA grading model

\begin{tabular}{|c|c|c|c|c|c|c|}
\hline & \multirow[t]{2}{*}{ Gleason grade (GG) } & \multicolumn{4}{|c|}{ Predicted group membership } & \multirow[t]{2}{*}{ Total } \\
\hline & & GG 2 & GG 3 & GG 4 & GG 5 & \\
\hline \multirow[t]{4}{*}{ Count } & GG 2 & 11 & 0 & 0 & 0 & 11 \\
\hline & GG 3 & 0 & 5 & 0 & 0 & 5 \\
\hline & GG 4 & 0 & 0 & 4 & 0 & 4 \\
\hline & GG 5 & 0 & 0 & 1 & 6 & 7 \\
\hline \multirow[t]{4}{*}{$\%$} & GG 2 & 100 & 0 & 0 & 0 & 100 \\
\hline & GG 3 & 0 & 100 & 0 & 0 & 100 \\
\hline & GG 4 & 0 & 0 & 100 & 0 & 100 \\
\hline & GG 5 & 0 & 0 & 14.3 & 85.7 & 100 \\
\hline
\end{tabular}

were entered into the FTIR-LDA grading model as an unknown group. The minimum Euclidean distance between the unknown spectrum to any of the predefined groups was used to diagnose the disease state of the prostate tumour biopsy and these results are shown in Table 2.

The following points were considered for presenting results in Table 2. (1) If the tissue specimen exhibited lesions that comprised more than one Gleason grade, which could not be independently sampled by FTIR microspectroscopy, the histologic area was designated by $\mathrm{Gx}_{1}+\mathrm{Gx}_{2}$ (where, $\mathrm{Gx}_{1}$ and $\mathrm{Gx}_{2}$ represent the first and second Gleason grades, respectively). (2) For tissue sections exhibiting a single pCA lesion, the FTIR-LDA score is derived from the two highest FTIR-LDA grades. (3) For biopsies in which two independent foci of pCA have been identified by histology (case numbers 7, 10, 11, and 19 in Table 2) and which can be sampled independently by FTIR microspectroscopy, the histologic Gleason score is the sum of the highest grade at each lesion. Similarly, the FTIR-LDA score is the sum of the highest FTIR-LDA grades obtained at each lesion. (4) If the model generates one FTIR-LDA grade in the tissue, then the FTIR-LDA grade is doubled to produce the FTIR-LDA score. Point 4 follows a rule used to assign Gleason scores in present histopathologic practice [1].

The scatter plot in Fig. 4 of Gleason score versus FTIR-LDA score demonstrates a significant association $(p=0.01)$ between scores produced from the malignant lesions of each of the blind-tested biopsies in Table 2. This correlation between tissue biochemistry and architecture exists despite the fact that $59 \%$ of the biopsies with known hormone status were hormone manipulated. To further evaluate the association between Gleason score versus FTIR-LDA score, we set "stringent-rule criteria." The stringent-rule criteria are as follows. (1) If the Gleason score consists of an area exhibiting $\mathrm{Gx}_{1}+\mathrm{Gx}_{2}$, then agreement to FTIR-LDA score is based on the allocation of spectra to FTIR-LDA grades that coincide with both $\mathrm{Gx}_{1}+\mathrm{Gx}_{2}$ and no other FTIRLDA grade. (2) If the histologic Gleason score is determined from the doubling of a single Gleason grade, then agreement to FTIR-LDA score results from the allocation of spectra to the equivalent FTIRLDA grade and no other. In the light of the these criteria we acknowledge that of the 40 individual tumour locations sampled by FTIR-microspectroscopy, a $20 \%$ (8 of 40) agreement of FTIR-LDA score to Gleason score was achieved, annotated from Table 2.

In Table 2 we observe 24 tumour locations that were assigned a single Gleason grade. Of these, 4 (17\%) tumour locations had a single FTIR-LDA grade that was identical to the single Gleason grade, 13

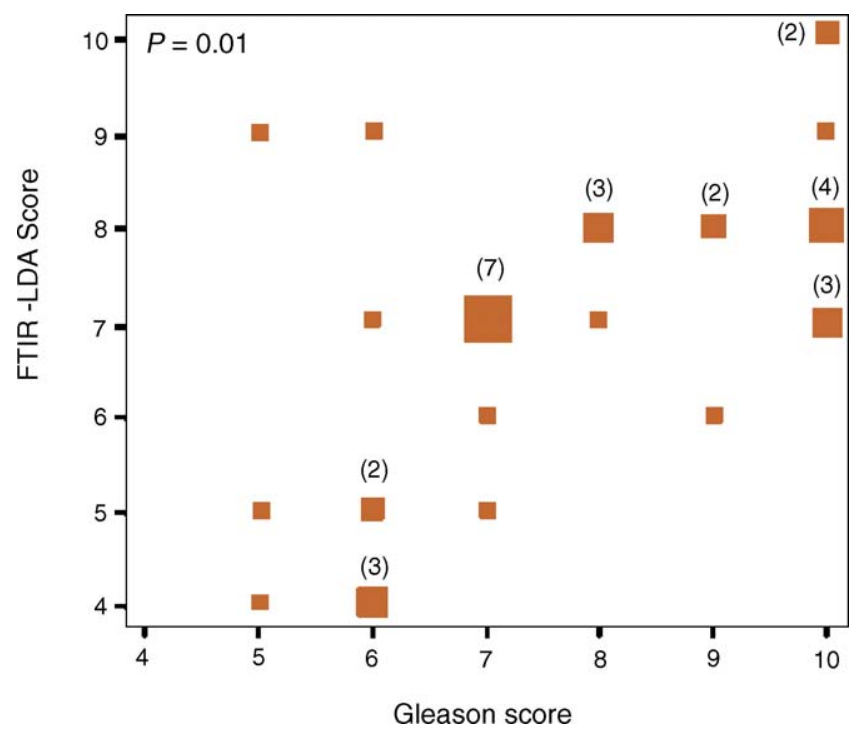

Fig. 4 - Correlation between Fournier transform infraredlinear discriminant analysis score with Gleason score for each of the biopsies tested in Table 2. The areas of the squares are proportional to the number of points at that location (in brackets). 
Table 2 - Summary of blind testing results for the FTIR-LDA tumour-grading model for prostate cancer

\begin{tabular}{|c|c|c|c|c|c|c|c|c|c|c|}
\hline \multirow[t]{2}{*}{$\begin{array}{l}\text { Case } \\
\text { no. }\end{array}$} & \multirow{2}{*}{$\begin{array}{l}\text { Histologic } \\
\text { Gleason } \\
\text { grade }\end{array}$} & \multirow{2}{*}{$\begin{array}{l}\text { Histologic } \\
\text { Gleason } \\
\text { score }\end{array}$} & \multirow{2}{*}{$\begin{array}{l}\text { Total no. of } \\
\text { IR spectra acquired } \\
\text { from specimen }{ }^{\mathrm{a}}\end{array}$} & \multicolumn{4}{|c|}{$\begin{array}{l}\text { FTIR-LDA } \\
\text { grade }^{b}\end{array}$} & \multirow{2}{*}{$\begin{array}{l}\text { FTIR- } \\
\text { LDA } \\
\text { score }^{c}\end{array}$} & \multirow{2}{*}{$\begin{array}{c}\text { Tumour } \\
\text { stage } \\
\text { (TNM system) }^{\mathrm{d}}\end{array}$} & \multirow[t]{2}{*}{$\begin{array}{c}\text { Hormone } \\
\text { manipulation }\end{array}$} \\
\hline & & & & 2 & 3 & 4 & 5 & & & \\
\hline 1 & $3+3$ & 6 & 4 & & & 2 & 2 & 9 & T1 Nx M1 & No \\
\hline 2 & $4+4$ & 8 & 4 & & & 4 & & 8 & T3 Nx M0 & No \\
\hline 3 & $4+4$ & 8 & 10 & & & 10 & & 8 & T3 Nx M0 & - \\
\hline 4 & $2+3$ & 5 & 9 & 5 & 4 & & & 5 & T1 Nx M0 & Yes \\
\hline 5 & $5+5$ & 10 & 6 & 1 & 3 & 2 & & 7 & - & - \\
\hline 6 & $5+5$ & 10 & 5 & & & & 5 & 10 & T4 Nx M0 & Yes \\
\hline \multirow[t]{2}{*}{7} & Lesion 1: $4+4$ & 9 & 8 & & 7 & 1 & & 8 & T3 Nx M0 & Yes \\
\hline & Lesion $2: 5+5$ & & 6 & & 3 & 3 & & & & \\
\hline 8 & $3+3$ & 6 & 11 & 11 & & & & 4 & T2 Nx M0 & - \\
\hline 9 & $5+5$ & 10 & 10 & & & 10 & & 8 & T4 Nx M0 & No \\
\hline \multirow[t]{2}{*}{10} & Lesion 1: $5+5$ & 10 & 11 & & & 11 & & 8 & T1b Nx M0 & No \\
\hline & Lesion $2: 5+4$ & & 6 & & & 6 & & & & \\
\hline \multirow[t]{2}{*}{11} & Lesion 1: $5+4$ & 10 & 12 & & 2 & 6 & 4 & 10 & T4 N1 M1 & - \\
\hline & Lesion $2: 5+5$ & & 5 & & & 3 & 2 & & & \\
\hline 12 & $5+5$ & 10 & 8 & & & 8 & & 8 & T1 Nx M1 & \\
\hline 13 & $3+3$ & 6 & 11 & 2 & 7 & 2 & & 7 & T3 Nx M0 & No \\
\hline 14 & $5+4$ & 9 & 10 & & & 10 & & 8 & T3 Nx M0 & Yes \\
\hline 15 & $2+3$ & 5 & 10 & 10 & & & & 4 & T1 NO MO & No \\
\hline 16 & $5+5$ & 10 & 14 & & & 14 & & 8 & T4 Nx M1 & Yes \\
\hline 17 & $3+4$ & 7 & 13 & & 10 & 3 & & 7 & T2 Nx M0 & - \\
\hline 18 & $2+3$ & 5 & 17 & & 6 & 8 & 3 & 9 & T3 Nx M0 & - \\
\hline \multirow[t]{2}{*}{19} & Lesion $1: 3+4$ & 9 & 10 & 2 & 8 & & & 6 & T1 N0 M1 & Yes \\
\hline & Lesion $2: 5+5$ & & 9 & 1 & 8 & & & & & \\
\hline 20 & $3+3$ & 6 & 8 & 8 & & & & 4 & T3 Nx MO & - \\
\hline 21 & $5+5$ & 10 & 10 & & 1 & 9 & & 7 & T4 Nx M0 & - \\
\hline 22 & $4+4$ & 8 & 10 & & & 10 & & 8 & T3 NO MO & Yes \\
\hline 23 & $3+3$ & 6 & 10 & 7 & 3 & & & 5 & T2 NO MO & Yes \\
\hline 24 & $3+3$ & 6 & 11 & 11 & & & & 4 & T1 Nx MO & Yes \\
\hline 25 & $5+5$ & 10 & 10 & & 5 & 5 & & 7 & T3 Nx MO & No \\
\hline 26 & $4+4$ & 8 & 10 & & 7 & 3 & & 7 & T3 N0 M1 & No \\
\hline 27 & $5+5$ & 10 & 13 & 2 & 3 & 7 & 1 & 9 & T3 Nx M1b & No \\
\hline 28 & $3+3$ & 6 & 10 & 4 & 6 & & & 5 & T2 Nx M0 & Yes \\
\hline 29 & $3+4$ & 7 & 12 & 11 & 1 & & & 5 & T1 Nx M0 & No \\
\hline 30 & $3+4$ & 7 & 10 & & 2 & 8 & & 7 & T3 Nx MO & No \\
\hline 31 & $4+3$ & 7 & 10 & 7 & & 3 & & 6 & T2 NO MO & Yes \\
\hline 32 & $3+4$ & 7 & 10 & 5 & 3 & 2 & & 7 & T1 NO MO & Yes \\
\hline 33 & $4+3$ & 7 & 10 & 5 & 4 & 1 & & 7 & T2 NO Mx & No \\
\hline 34 & $3+4$ & 7 & 10 & 1 & 4 & 5 & & 7 & T2 NO MO & Yes \\
\hline 35 & $4+3$ & 7 & 10 & 1 & 8 & 1 & & 7 & T3 Nx M0 & Yes \\
\hline 36 & $4+3$ & 7 & 10 & & 8 & 2 & & 7 & - & Yes \\
\hline
\end{tabular}

FTIC-LDA = Fournier transform infrared-linear discriminant analysis.

a The numbers reported in this field are dependent on the size of the tumour lesion within the tissue section as well as positive identification of tumour glands in the unstained tissue section.

$\mathrm{b}$ The numbers reported in this field represent the number of individual IR spectra (derived from different locations within the specimen) classified by the model to a particular cluster.

c The FTIR-LDA score is derived from the sum of the two highest FTIR-LDA grades to which the individual IR spectra allocate.

d A dash is used to indicate where staging data was unavailable.

e Patients undergoing hormone manipulation by either surgical or chemotherapeutic treatment. A dash is used to indicate where hormone status was unavailable.

(54\%) locations had all/some IR spectra allocating to FTIR-LDA grades that deviated by one grade lower and/or higher than to the Gleason grade, and one specimen (Table 2, case number 19, lesion 2) deviated by more than one grade compared to the Gleason grade. Finally, 6 (25\%) locations had FTIRLDA grades that deviated by more than one grade (higher or lower) compared with the Gleason grade.
However, in these last 6 locations, deviation by more than one FTIR-LDA grade from the Gleason grade was accompanied with an FTIR-LDA grade that deviated by only one grade from the Gleason grade.

Table 3 shows the sensitivity and specificity obtained by the FTIR-LDA scoring model to classify biopsies according to the three-band Gleason criterion [5,15-17]. This method is a more clinically 
Table 3 - Sensitivity and specificity for classification of prostate tumour biopsies by FTIR-LDA according to the three-band Gleason criterion

\begin{tabular}{lccc}
\hline & Gleason score $<7$ & Gleason score $=7$ & Gleason score $>7$ \\
\hline Total no. of spectra (no. of biopsies) & $101(10)$ & $95(9)$ & $187(17)$ \\
Sensitivity & $70 \%$ & $78 \%$ & $71 \%$ \\
Specificity & $89 \%$ & $81 \%$ & $89 \%$ \\
\hline FTIR-LDA = Fournier transform infrared-linear discriminant analysis. & & \\
\hline
\end{tabular}

orientated scale in which Gleason scores of $<7$ are considered to be relatively less aggressive tumours than those that exhibit Gleason scores $=7$. The latter is considered to be less aggressive than Gleason

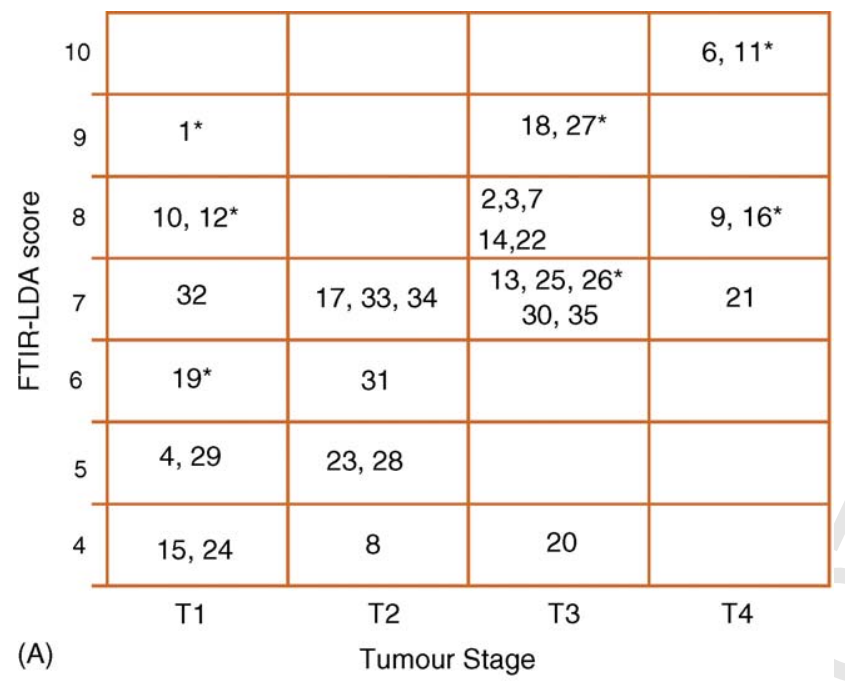

\begin{tabular}{|c|c|c|c|c|}
\hline \multirow{5}{*}{ 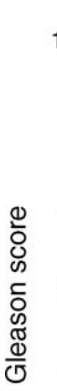 } & $10,12^{*}$ & & $25,27^{\star}$ & $\begin{array}{l}6,9,11^{\star} \\
21,16^{\star}\end{array}$ \\
\hline & $19^{*}$ & & 14,7 & \\
\hline & & & $\begin{array}{l}2,3 \\
22,26^{*}\end{array}$ & \\
\hline & 29,32 & $\begin{array}{l}17,31 \\
33,34\end{array}$ & 30,35 & \\
\hline & $1^{\star}, 24$ & $8,23,28$ & 13,20 & \\
\hline 5 & 4,15 & & 18 & \\
\hline 4 & & & & \\
\hline & $\mathrm{T} 1$ & T2 & Т3 & $\mathrm{T} 4$ \\
\hline (B) & & Tumou & & \\
\hline
\end{tabular}

Fig. 5 - Charts showing the correlation between (A) Fournier transform infrared-linear discriminant analysis score and (B) Gleason score with tumour stage at time of biopsy. In each chart, the numbers displayed relate to case numbers presented in Table 2. The case numbers designated with an asterisk represent metastatic tumours, those without an asterisk represent nonmetastatic tumours. scores of $>7$, in which a high risk of metastasis is more likely at the time of diagnosis. In Table 3, we observe a sensitivity of $\geq 70 \%$ for the FTIR-LDA system to predict Gleason score, based on the threeband criteria for each biopsy tested, with specificity $\geq 81 \%$.

3.3. Correlating FTIR-LDA score to the clinical stage of the prostatic tumour

Of the 36 biopsy specimens used for blind testing in this study, the clinical stage (using the TNM system of prostate tumour staging) pertaining to 31 of these biopsies was available (Table 2). Fig. 5 illustrates the correlation of FTIR-LDA and Gleason score with the clinical stage of the tumour at time of biopsy. The FTIR-LDA and Gleason scores derived from T1-T2 stage tumours were significantly different ( $p=0.004)$ from FTIR-LDA scores of T3-T4 stage tumours. More specifically, $93 \%$ of $\mathrm{T} 1-\mathrm{T} 2$ stage tumours with no metastases provide FTIR-LDA scores of $\leq 7$ and Gleason scores of 5-7. For advanced tumours of T3-T4 stage, with no metastases, $62 \%$ and $6 \%$ of these tumours give rise to FTIR-LDA and Gleason scores of $\geq 8$, respectively.

Using a Gleason/FTIR-LDA score of $\geq 8$ as a cut-off point to predict metastatic tumours, we obtain a sensitivity and specificity of $71 \%$ and $67 \%$, respectively, for the prediction of metastatic tumours using the FTIR-LDA system. Using the same cut-off point, we obtain specificity and sensitivity of $85 \%$ and $63 \%$, respectively, for the prediction of metastatic tumours using the Gleason system.

\section{Discussion}

\subsection{Construction of the FTIR-LDA pCA tumour-grading model}

In the present study a single experienced histopathologist (J.H.S.) assigned Gleason scores to tissue sections of pCA. This was carried out because a study by Lattouf et al. [3] reports $29.2 \%$ agreement in assignment of Gleason score to biopsy and radical prostatectomy samples when assessed by up to 15 
pathologists. However, the same study showed that when samples were assessed by a single pathologist, the agreement rose to $48.7 \%$. They conclude that assessment by the same pathologist reduces the discrepancy in the Gleason grading of prostate biopsy. Nevertheless, the variability in assigning a Gleason grade to any given area of pCA tissue, even by a single pathologist as outlined by Lattouf et al. [3], complicates the establishment of suitable reference standards to train the FTIR-LDA-grading model. Therefore, clinical records were obtained for the tumours from which each of the training Gleason-graded pCA tissue specimens were derived.

The Gleason grade 2 training specimen originated from a clinically low-stage tumour (T2NOM0), whereas, the Gleason grade 4 and 5 training specimens were derived from tumours that exhibited bone metastases at time of biopsy. Interestingly, the Gleason grade 4 and Gleason grade 5 clusters in the FTIR-LDA-grading model (Fig. 3A) are in closer proximity to one another than any of the other groups in the template. With correlation to the clinical data presented, we can postulate that because both tumours are highly aggressive, they may consist of cells with overlapping phenotypes, which result in overlapping spectral features in their associated IR spectra. This is highlighted by the fact that one IR spectrum from the Gleason grade 5 group classifies to the Gleason grade 4 group in the initial classification results of this FTIR-LDA grading model (Table 1).

The Gleason grade 3 training specimen was derived from a patient whose tumour exhibited bone metastases at time of biopsy. However, the projections of IR spectra acquired from this specimen onto the FTIR-LDA grading model (Fig. 3A) are well separated from the Gleason grade 4 and 5 clusters. Thus, it may be the case that the proportion of malignant cells sampled by FTIR, from the total population of malignant cells in this Gleason grade 3 specimen, are not as aggressive as those sampled from the Gleason grade 4 and 5 specimens. It is well known that tumours of a particular Gleason grade can exhibit intratumour and intertumour phenotypic and genotypic heterogeneity [18].

\subsection{Correlating biospectroscopic FTIR-LDA score to histologic Gleason score}

The low correlation (20\%) of biospectroscopic FTIRLDA score with histologic Gleason score, using the stringent-rule criteria of evaluation, is not entirely unexpected because Gleason grading is based solely on tissue architecture, whereas the direct variable entered into the LDA algorithm is associated with spectroscopic differences that reflect the biochemical composition from one disease state compared with another. Furthermore, using the stringent-rule criteria we compared the difference in FTIR-LDA grades with Gleason grade for 24 tumour lesions assigned with a single Gleason grade. This yielded two important results. First, only $17 \%$ of the 24 tumour locations analysed by FTIR-LDA agreed with histology in assigning a single grade, which suggests that the FTIR-LDA model identifies greater biochemical heterogeneity in the population of pCA cells, within these tumours sections, than is evident through visual (Gleason) grading of tissue architecture. Second, this heterogeneity in biochemistry within a single focus of PCA is identified by the FTIRLDA model as a continuum of biochemical changes between each successive phase of pCA progression. This result is exemplified by six locations, which produced FTIR-LDA grades that differed by two grades from the corresponding Gleason grade, but was accompanied by a difference of one FTIR-LDA grade.

We investigated the association between FTIRLDA score with Gleason score in terms of the threeband Gleason criterion, which divided specimens into groups corresponding to Gleason scores $<7,=7$, and $>7$. We chose to subdivide the Gleason system in this manner rather than into groups corresponding to scores 2-3-4, 5-6-7, and 8-9-10, because Oesterling et al. [19] and Gleason [20] report that the individual scores that constitute the central group of tumours (5-6-7), behave very differently. A detailed analysis of the sensitivity and specificity achieved for the FTIR-LDA model to predict Gleason score is discussed in Section 4.3.

4.3. Correlating FTIR-LDA score to the clinical stage of the prostatic tumour

The distinction between T1-T2 and T3-T4 tumours is important, because it is generally accepted that the presence of capsular penetration, invasion to surrounding anatomy and systemic metastases, diminishes the probability of a cure. Encouragingly, we find the FTIR-LDA and Gleason scores of tumours with stage T1-T2 were statistically different from tumours with stage T3-T4 disease. Note, that this differentiation exists despite the fact $59 \%$ of our blind-tested specimens are derived from hormonemanipulated tumours (Table 2).

Two biopsies from our blind test cohort were stage T3 tumours with Gleason score 7 (Table 2 or Fig. 5B, cases 30 and 35) and it is well known that tumours of this type demonstrate variability with regard to disease progression. Both of these tumours 
396 gave rise to an FTIR-LDA score of equal value (7). 397 Other biopsies obtained from stage T3 tumours, $398 \quad$ which score 7 by the FTIR-LDA model (Table 2 or Fig. 5A, cases 25 and 26), score highly using the Gleason system (8-10), with case 26 originating from a metastatic tumour and case 25 developing skeletal metastases. Follow-up data revealed that case 35 originated from a hormone naive tumour, whereas for case 30 the tumour had progressed to metastatic disease. A larger number of biopsies with follow-up information will be required to validate to what extent an FTIR-LDA score of 7 may predict the propensity for disease progression.

In Section 4.2, the stringent-rule criteria for the evaluation of the FTIR-LDA system revealed that for the majority of tumour lesions, a greater heterogeneity in disease state was identified when compared to Gleason grading. This result is of particular importance if the tumour lesion provides an FTIR-LDA grade that is higher than the assigned histologic grade. This could indicate malignant cells that have developed more aggressive phenotypes before the onset of tissue architectural change. The FTIR-LDA system achieved $70 \%$ sensitivity to predict Gleason score $<7$ because 1 (case 13) and 2 (cases 1 and 18) of 10 biopsies classified to the Gleason score 7 and Gleason score $>7$ bands, respectively. From Table 2, we find that case 1 was assigned a Gleason score of $6(3+3)$ and an FTIR-LDA score of $9(5+4)$. On further analysis, case 1 (high FTIR-LDA score) was derived from a prostate tumour exhibiting bone metastases at time of biopsy. Similarly, case 18 was assigned a Gleason score of $5(3+2)$ and an FTIR-LDA score of $9(5+4)$. Although $35 \%$ of IR spectra derived from case 18 allocated to the grade 3 group, the two highest FTIRLDA grades were summed to provide the FTIR-LDA score for this tumour section. It was found that the biopsy from case 18 also originated from a prostate tumour that exhibited aggressive behaviour because it presented with extracapsular penetration. Thus, for cases 1 and 18, a higher FTIR-LDA score than the associated Gleason score, was consistent with the more aggressive phenotypic behaviour evident as aggressive local and distant spread of tumours in these patients. Finally, case 13 was assigned a Gleason score of $6(3+3)$, an FTIR-LDA score of 7 $(3+4)$ and a clinical stage of T3NxM0. The higher FTIR-LDA score for case 13 is also consistent with the aggressive nature of this tumour.

Equally important in identifying underscored tumours are those that are overscored using the Gleason system. In Table 3 we observe that for Gleason score $=7$, we achieve $78 \%$ sensitivity for the FTIR-LDA predictive score. This is because two cases
(Table 2, cases 29 and 31) of nine Gleason score $=7$ biopsies classified to the FTIR-LDA score $<7$ band and were of T1NxM0 and T2NxM0 staged tumours. Thus, for these Gleason score $=7$ biopsies, a low FTIR-LDA score reinforces the low stage of these tumours. Although, the biopsies for cases 32 and 33 produce Gleason and FTIR scores of 7 for these T1 and T2 stage tumours, $\geq 80 \%$ of the total number of IR spectra collected from these tumours correlated to FTIR-LDA grades of 2 and 3 (Table 2). Similarly, for case 17, which gave rise to a Gleason and FTIR-LDA score of 7 for this T2 stage tumour, $77 \%$ of the total number of spectra collected from this specimen correlated to an FTIR-LDA grade of 3 . Nevertheless, we use the two highest grades to determine the FTIR-LDA score. Consequently, the FTIR-LDA model may have differentiated less aggressive Gleason 7 tumours (cases 29 and 31) from those that may be on the onset of $\mathrm{T} 3$ stage (cases $32,33,17$ ). This is supported by case 30 , which was assigned an FTIR-LDA and Gleason grade 7 , but which was observed to demonstrate a shift in the proportion of spectra (80\%) to the FTIR-LDA grade 4 group, for this T3 stage tumour.

Recently, Crow et al. [7] have integrated Raman spectroscopy with principle component fed LDA to develop and blind test a diagnostic tool for pCA grading, in vitro, based on the three-band Gleason criterion. The sensitivity and specificity obtained by Crow et al. were slightly higher than those obtained in our FTIR-based study. However, whether the higher sensitivity and specificity achieved in the Ramanbased study was beneficial in terms of predicting tumour biopotential remains to be assessed. Furthermore, the method used to evaluate the performance of the Raman- based model was the leave-one-out (cross-validation) procedure. Although the authors use a number of biopsies for the construction of their diagnostic algorithms, multiple spectra from the same biopsies were acquired. Therefore, the leaveone-out procedure contains a degree of bias because the dataset the retained spectrum is blind tested against has already incorporated spectral patterns (the linear combination of predictor variables) from the same sample.

The FTIR-LDA model gave rise to a slightly lower sensitivity $(-14 \%)$ for correlation with metastases, when compared with the Gleason system. However, it did provide slightly higher specificity $(+4 \%)$, indicating that the probability of the FTIR-LDA model to incorrectly predict aggressive metastatic behaviour in an individual patient without metastases is lower when compared with the Gleason system. An FTIR-based study by Malins et al. used pCA in conjunction with logistic regression to predict the probability for metastasis at $\sim 90 \%$ sensitivity and 
specificity using primary prostate tumour DNA derived from patients who exhibited metastases at time of biopsy [8]. However, DNA extraction methods require careful macrodissection of tissue zones enriched with malignant cells and the availability of frozen tissue. Thus, it is important to apply FTIRmicrospectroscopic diagnosis to paraffin-embedded tissue samples, which make up the majority of the presently archived database.

It is important to note that in our study, analysis of only one section per biopsied tumour was conducted. In current histopathologic practices, several sections are acquired from the same tumour to provide a better representation of the disease in the whole tumour. Hence, interpretation of our results must be considered with regard to this limitation. However, a tissue microarray system has recently been developed for high-throughput FTIR analysis of prostate tissue sections by Fernandez et al. [12]. Although the authors [12] did not attempt to stratify the severity of disease in malignant lesions, the feasibility of imaging FTIR microspectroscopy to differentiate different tissue components such as nerves, blood, and lymphocytes as well as benign from malignant prostatic epithelium was demonstrated. Thus, the results presented in this study, together with the potential for high-throughput screening, reported by Fernandez et al., clearly demonstrate the enormous potential for the classification of malignant prostatic tumours through IR biospectroscopy. Further information concerning improvements required to the FTIR technology before it is acceptable for clinical practice is reported in reference [21].

\section{Conclusions}

The association between FTIR-LDA score with Gleason score, for each of the biopsies tested in the blind study, was significant. However, the stringent-rule criteria were proposed so that the differences between FTIR-LDA grades compared with Gleason grades, for each biopsy tested in the blind study, could be assessed with higher precision. Using this method of evaluation, a $20 \%$ agreement of FTIR-LDA score to histologic score was obtained. An important conclusion as a result of the stringentrule criteria is that the FTIR-LDA scoring model represents a continuum of biochemical changes between each successive stage of pCA progression. The three-band Gleason criteria provided greater correlation of FTIR-LDA grade to histologic grade with $\geq 70 \%$ sensitivity and $\geq 81 \%$ specificity.

FTIR-LDA scores generated from biopsies of T1$\mathrm{T} 2$ and T3-T4 stage tumours were significantly different and this difference was independent of whether the tumour was sampled following hormone manipulation. Additionally, we found evidence confirming the beneficial utility of FTIR-LDA scoring in the identification of tumours that were more or less aggressive than their corresponding Gleason score suggested. This is particularly so for the Gleason score 7 biopsies analysed by FTIR-LDA in this study. Finally, we found that the Gleason and FTIR-LDA systems performed similarly in predicting metastatic tumours, using a cut-off point of $\geq 8$ for association with metastatic disease.

In conclusion, molecular diagnosis using the FTIR-LDA system has the potential to provide significant value in improving histologic assessment and prediction of the natural history of pCA. The evidence presented in this report is sufficient to warrant the further testing of this methodology on a larger dataset of patients.

\section{Acknowledgements}

We acknowledge EPSRC and the AICR for financial support (E. Gazi). We gratefully thank Mr. Gary Ashton and Miss Caron Abbey (Cancer Research UK, Paterson Institute, Manchester) for the preparation of histologic sections.

\section{References}

[1] Gleason DF. Histologic grading and clinical staging of prostatic carcinoma. In: Tannenbaum M, editor. Urological pathology - the prostate. Philadelphia, PA: Lee \& Febiger; 1977. p. 171-97.

[2] Tetu B. Morphologic changes induced by neoadjuvant combination hormone therapy on prostatic tissue and prostate cancer. Endocr Relat Cancer 1996;3:165-70.

[3] Lattouf JB, Saad F. Gleason score on biopsy: is it reliable for predicting the final grade on pathology? BJU Int 2002; 90:694-9.

[4] Smith EB, Frierson HF, Millis SE, Boyd JC, Teodorscu D. Gleason scores of prostate biopsy and radical prostatectomy specimens over the past 10 years. Is there evidence of systemic upgrading? Cancer 2002;94:2282-7.

[5] Epstein JI, Pizov G, Walsh PC. Correlation of pathological findings with progression after radical retropubic prostatectomy. Cancer 1993;71:3582-93.

[6] Levin IW, Bhargava R. Fourier transform infrared vibrational spectroscopic imaging: integrating microscopy and molecular recognition. Annu Rev Phys Chem 2005;56:429-74.

[7] Crow P, Stone N, Kendall CA, et al. The use of Raman spectroscopy to identify and grade prostatic adenocarcinoma in vitro. Br J Cancer 2003;89:106-8. 
[8] Malins DC, Johnson PM, Wheeler TM, Barker EA, Polissar NL, Wheeler TM. Cancer-related changes in prostate DNA as men age and early identification of metastases in primary prostate tumours. Proc Natl Acad Sci USA 2003; 100:5401-6.

[9] Bertucci F, Viens P, Tagett R, Nguyen C, Houlgatte R, Birnbaum D. DNA arrays in clinical oncology: promises and challenges (minireview). Lab Invest 2003;83:305-16.

[10] Gazi E, Dwyer J, Gardner P, et al. Applications of FTIRmicrospectroscopy to benign prostate and prostate cancer. J Pathol 2003;201:99-108.

[11] Gazi E, DwyerJ, Lockyer NP, et al. The combined application of FTIR microspectroscopy and ToF-SIMS imaging in the study of prostate cancer. Faraday Discuss 2004;126:41-59.

[12] Fernandez DC, Bhargava R, Hewitt SM, Levin IW. Infrared spectroscopic imaging for histopathologic recognition. Nat Biotechnol 2005;23:469-74.

[13] Stuart B. Instrumentation. Biological applications of infrared spectroscopy. London, United Kingdom: John Wiley \& Sons; 1997,, 26 p.

[14] Discriminant function analysis. In: Tabachnick, B.G., Fidell, L.S., editors. Using multivariate statistics, ed. 4. London, United Kingdom: Pearson; 2001. p. 456-61.
[15] Zagars GK, Pollack A, vonEschenbach AC. Prognostic factors for clinically localized prostate carcinoma-analysis of 938 patients irradiated in the prostate specific antigen era. Cancer 1997;79:1370-80.

[16] Coetzee LJ, Layfield LJ, Hars V, Paulson DF. Proliferative index determination in prostatic carcinoma tissue: is there any additional prognostic value greater than that of Gleason score, ploidy and pathological stage? J Urol 1997;157:214-8.

[17] Benaim EA, Pace CM, Roehrborn CG. Gleason score predicts androgen independent progression after androgen deprivation therapy. Eur Urol 2002;42:12-7.

[18] Lapointe J, Li C, Higgins JP, et al. Gene expression profiling identifies clinically relevant subtypes of prostate cancer. Proc Natl Acad Sci USA 2004;101:811-6.

[19] Osterling JE, Brendler CB, Epstein JI, Kimball AW, Walsh PC. Correlation of clinical stage, serum prostatic acid phosphatase and preoperative Gleason grade with final pathologic stage in 275 patients with clinically localized adenocarcinoma of the prostate. J Urol 1987;138:92-8.

[20] Gleason DF. Histologic grading of prostate cancer: a perspective. Hum Pathol 1992;23:273-9.

[21] General discussion. Faraday Discuss 2004; 126:77-92.

\section{Editorial Comment}

H.G. van der Poel, Department Urology,

Netherlands Cancer Institute, Amsterdam,

The Netherlands

h.vd.poel@nki.nl

The histological diagnosis of prostate cancer has been a field of improvement in recent years. Several immunohistochemical markers have been added to the pathologists armamentarium. In 2002 beschreven Rubin et al. [1] alpha-methylacyl coenzyme A racemase (AMACR) overexpression in prostate cancer and this marker is now often used, in combination with a basal-cell cytokeratin marker or p63 to aid in the differentiation of malignant or proliferative abnormalities in the prostate [2]. Despite these important recent findings and multiple attempts no widely excepted immunohistochemical analyses are available to predict tumor behaviour better than Gleason grade.

IHC analysis has hinted to specific molecular alterations in the development and progression of prostate cancer. Despite the fact that a large number of studies showed correlation of a variety of molecular markers with outcome after prostatectomy and local radiotherapy none of the markers is currently widely used for staging purposes. An important problem is the poor correlation between biopsy material results and prostatectomy histology suggesting inadequate tumor sampling using transrectal biopsies [3]. The often observed intratumoral heterogeneity in IHC staining further compromises accurate molecular staging.

Infrared microspectroscopy may be helpful to document structural and chemical composition of tissue and may supplement the information obtained by visual light microscopy but will still be hampered by the presence of tumor heterogeneity. Although the spectroscopy information initially was only a crude reflection of tissue composition further mathematical interpretations of the obtained infrared spectra has been suggested to aid in for example the spectrosopical identification of adenocarcinoma of the colon.

Gazi et al. present here one of the first steps of integration of this technology in the classification of prostate cancer and found reasonable correlation with visual Gleason grading and metastases prediction. Unfortunately, the heterogeneity of their study population prohibits conclusions regarding postoperative survival. Since in the presented population almost half of the patients received hormonal treatment the influence of hormonal manipulations on spectroscopy results should be more carefully considered.

More extensive analysis of properly selected populations is required to conclude on the value of this new technology. The identification of cancer in biopsy material or the correlation between spectroscopy and specific genetic alterations such as PTEN loss and Bcl-2 overexpression may be such studies. 


\section{References}

[22] Rubin MA, Zhou M, Dhanasekaran SM, Varambally S, Barrette TR, Sanda MG, et al. alpha-Methylacyl coenzyme A racemase as a tissue biomarker for prostate cancer. JAMA 2002;287:1662-70.

[23] Jiang Z, Li C, Fischer A, Dresser K, Woda BA. Using an AMACR (P504S)/34betaE12/p63 cocktail for the detec- tion of small focal prostate carcinoma in needle biopsy specimens. Am J Clin Pathol 2005;123:231-6.

[24] Stackhouse GB, Sesterhenn IA, Bauer JJ, Mostofi FK, Connelly RR, Srivastava SK, et al. p53 and bcl-2 immunohistochemistry in pretreatment prostate needle biopsies to predict recurrence of prostate cancer after radical prostatectomy. J Urol 1999;162: 2040-5. 